Research Paper

\title{
Synovial Cell Count Poorly Predicts Septic Arthritis in the Presence of Crystalline Arthropathy
}

\author{
T. David Luo ${ }^{1 * \bowtie}$, D. Landry Jarvis ${ }^{2 *}$, Hunter B. Yancey ${ }^{1}$, Andrey Zuskov ${ }^{1}$, Shane C. Tipton ${ }^{1}$, Maxwell K. \\ Langfitt ${ }^{1}$, Johannes F. Plate \\ 1. Department of Orthopaedic Surgery, Wake Forest Baptist Medical Center, Winston-Salem, NC 27157, USA \\ 2. Divison of Sports Medicine, Department of Orthopaedic Surgery, Duke University Medical Center, Durham, NC 27710, USA \\ ${ }^{*}$ T. David Luo and D. Landry Jarvis, as co-first authors, contributed equally to this study. \\ $\square$ Corresponding author: T. David Luo, MD, Medical Center Blvd, Winston-Salem, NC 27157. Ph: 336-713-4025; Fax: 336-716-7310; Email: tluo@wakehealth.edu \\ ( ) The author(s). This is an open access article distributed under the terms of the Creative Commons Attribution License (https://creativecommons.org/licenses/by/4.0/). \\ See http://ivyspring.com/terms for full terms and conditions.
}

Received: 2020.02.11; Accepted: 2020.03.08; Published: 2020.04.22

\begin{abstract}
Introduction: A synovial cell count greater than $50,000 / \mathrm{mm}^{3}$ is the threshold most commonly used to diagnose septic arthritis. This lab value may be nonspecific in the setting of crystalline arthropathy. The purpose of this study was to evaluate the accuracy of diagnosing septic arthritis using a synovial cell count cut-off of $50,000 / \mathrm{mm}^{3}$ in the setting of crystalline arthropathy.

Methods: This was a retrospective review of joint aspirations performed between July $1^{\text {st }}, 2013$ and June $30^{\text {th }}$, 2016. Synovial fluid samples were evaluated for cell count, crystals, Gram stain, and culture. The sensitivity, specificity, positive predictive value (PPV), and negative predictive value (NPV) of the synovial markers were calculated.

Results: During the study period, 738 joint aspirations were sent for testing, of which 358 aspirations in 348 patients met inclusion criteria. There were $49(13.7 \%)$ cases of culture-positive septic arthritis, and 47 patients underwent surgical irrigation and debridement. Gout and pseudogout crystals were present in 163 aspirates (45.5\%). Three joints ( $0.8 \%$ overall rate) had concomitant crystalline arthropathy and septic arthritis, each of which had a synovial WBC $\geq 85,000 / \mathrm{mm}^{3}$. Increasing the WBC count cutoff to $85,000 / \mathrm{mm}^{3}$ demonstrated a specificity of $100 \%$, but a PPV of $12.0 \%$.

Conclusions: A cut-off of $85,000 / \mathrm{mm}^{3}$ may be more appropriate to diagnose concomitant septic arthritis and crystalline arthropathy. We recommend medical management and observation in patients with crystal-positive joint aspirations unless the synovial cell count is elevated above $85,000 / \mathrm{mm}^{3}$. Prospective studies using this treatment guideline are needed to evaluate its validity and accuracy.
\end{abstract}

Key words: septic arthritis, crystalline arthropathy, gout, pseudogout, cell count, joint infection

\section{Introduction}

Septic arthritis was found to be responsible for 16,382 emergency room visits in the US in 2012, of which $83 \%$ required hospital admission, resulting in a significant financial burden to the healthcare system. ${ }^{1}$ Septic arthritis of a native joint is considered one of the few orthopaedic emergencies. The presence of bacteria inside a joint can lead to cartilage degeneration within days of onset if not properly diagnosed and treated. ${ }^{2}$ The diagnosis of septic arthritis, however, can be challenging, as there are many other less urgent pathologies such as osteoarthritis, crystalline arthropathy, autoimmune disorders, viral infections, and trauma that mimic the presentation of a red, swollen, painful joint.3,4 
The gold standard for the diagnosis of septic arthritis is arthrocentesis and culture of the synovial fluid. A synovial white blood cell count (WBC) greater than $50,000 / \mathrm{mm}^{3}$ is highly indicative of septic arthritis and is the typical threshold used by most orthopaedic surgeons to determine the patient's need for surgical irrigation and debridement (I\&D). ${ }^{5-7}$ The synovial cell count cut-off of $50,000 / \mathrm{mm}^{3}$, however, has been shown to lack sensitivity for septic arthritis. ${ }^{6}$ In the setting of crystalline arthropathy (gout or pseudogout), the synovial cell count may be further elevated.6,8,9 With the reported co-existence of active gout in a septic joint ranging from $<2 \%$ to $23 \%$, interpreting synovial cell count and making the correct diagnosis can be difficult. $8,10,11$

While most surgeons interpret a synovial WBC $\geq 50,000 / \mathrm{mm}^{3}$ and negative crystals as a septic joint until proven otherwise, managing a patient with, for example, a synovial WBC count of $65,000 / \mathrm{mm}^{3}$ with negatively-birefringent crystals can be much more ambiguous. There is no consensus on the treatment of this hypothetical patient, and limited literature exists for this clinical scenario. Some surgeons may emergently take the patient to the operating room, while others may adhere to the gout diagnosis and recommend medical management, while awaiting the final results of the bacterial culture from the arthrocentesis. The purpose of this study was to evaluate the effect of crystalline arthropathy on the accuracy of diagnosing septic arthritis in a native joint with respect to the use of a synovial cell count cut-off of $50,000 / \mathrm{mm}^{3}$ and to establish guidelines for surgeons in this scenario. We hypothesized that crystalline arthropathy leads to increased synovial fluid cell count and thereby increased number of false positive diagnoses of septic arthritis and unnecessary surgical I\&D's.

\section{Methods}

This study was a retrospective chart review of joint aspirations performed at a Level 1 tertiary academic hospital between July 1st, 2013 and June 30th, 2016. All aspects of the study were approved by our Institutional Review Board (IRB00041702). The inclusion criteria were any adult patient ( $\geq 18$ years of age) who underwent synovial aspiration of a native hip, knee, ankle, shoulder, elbow, wrist, metacarpophalangeal or metatarsophalangeal joint in either the Emergency Department or inpatient setting. Patients with prior surgery to the joint evaluated for septic arthritis were also excluded.

For the patients who met inclusion criteria, demographic data (age, sex), past medical history (diabetes, immunocompromising diseases or medications, smoking status, previous antibiotic exposure), clinical presentation (temperature, presence of joint effusion on plain radiograph), blood serum markers [WBC, absolute neutrophil count (ANC), C-reactive protein (CRP), erythrocyte sedimentation rate (ESR)], and synovial fluid markers [WBC count with polymorphonuclear lymphocyte (PMN) percentage, crystals, Gram stain, and culture] were assessed. Any samples that were of insufficient volume $(<1 \mathrm{~mL})$ to evaluate these laboratory parameters were excluded. Cell count was performed manually within one hour of obtaining the synovial samples by our institution's microbiology laboratory. The presence of crystals was detected using polarized light microscopy.

A joint was defined to have gout or pseudogout based on the presence of negatively- or positively-birefringent crystals in the joint aspirate, respectively. For analysis purposes, both gout and pseudogout were grouped under the umbrella of "crystalline arthropathy". A joint was defined to be septic based on the presence of bacteria from the culture of the initial joint aspiration. Also, if the initial aspirate was culture-negative, but a synovial culture obtained during a subsequent surgical I\&D was positive, then these joints were also categorized in the septic group. Culture contamination is a relatively common occurrence, most often due to the ubiquitous skin organism coagulase-negative Staphylococcus (CoNS), which is a rare pathogen in native septic arthritis. ${ }^{12}$ Any aspirate positive for CoNS was regarded a true septic joint only if this was reinforced with a second positive culture obtained intraoperatively. Otherwise, if the patient was treated successfully without surgical I\&D, if subsequent intraoperative cultures were negative, or if the clinical picture was not consistent with septic arthritis based on orthopaedic evaluation and infectious disease consultation, the aspirate was categorized in the aseptic control group.

\section{Statistical Analysis}

Categorical variables were assessed using chi-square and Fisher exact tests. Univariate and multivariate analyses were performed to assess patient characteristics and risk factors. Accuracy of synovial WBC count or Gram stain to diagnose septic arthritis was calculated as "(true positive + true negative) / total number of aspirates" and illustrated using receiver operating characteristic (ROC) curves. The sensitivity, specificity, positive predictive value (PPV), and negative predictive value (NPV) were calculated. Subgroup analysis comparing aspirates with and without crystals was also performed. Data are represented in mean \pm standard deviation (SD) and $95 \%$ confidence intervals (CI). A p-value of 0.05 was used to define statistical significance. 
Table 1. Patient characteristics of 358 aspirations performed in 348 patients. Each aspiration was treated as a unique data point.

\begin{tabular}{lll}
\hline & N & $\%$ \\
\hline Sex & & \\
Male & 231 & $64.5 \%$ \\
Female & 127 & $35.5 \%$ \\
Joint & & \\
Knee & 230 & $64.3 \%$ \\
Ankle & 38 & $10.6 \%$ \\
Wrist & 24 & $6.7 \%$ \\
Elbow & 23 & $6.4 \%$ \\
Hip & 21 & $5.9 \%$ \\
Shoulder & 16 & $4.5 \%$ \\
Metatarsophalangeal & 5 & $1.4 \%$ \\
Metacarpophalangeal & 1 & $0.3 \%$ \\
Laterality & & \\
Left & 176 & $49.2 \%$ \\
Right & 182 & $50.8 \%$ \\
Aspiration performed by & & \\
Orthopaedic Surgery & 159 & $44.4 \%$ \\
Emergency Department & 158 & $44.1 \%$ \\
Interventional Radiology & 23 & $6.2 \%$ \\
Internal Medicine & 9 & $2.5 \%$ \\
Rheumatology & 8 & $2.2 \%$ \\
Plastic Surgery & 2 & $0.6 \%$ \\
\hline
\end{tabular}

Table 2. Microbiology results of the 49 cases of septic arthritis.

\begin{tabular}{lll}
\hline Staphylococcal species & $\mathbf{N}=\mathbf{3 3}$ & $\mathbf{6 7 . 3} \%$ \\
MSSA & 21 & $42.9 \%$ \\
MRSA & 9 & $18.3 \%$ \\
CoNS & 3 & $6.1 \%$ \\
Streptococcus species & $\mathbf{N}=\mathbf{4}$ & $\mathbf{8 . 2} \%$ \\
Group A Streptococcus (GAS) & 1 & $2.0 \%$ \\
Group B Streptococcus (GBS) & 2 & $4.1 \%$ \\
Group C Streptococcus (GCS) & 1 & $2.0 \%$ \\
Pseudomonas & 5 & $10.2 \%$ \\
Escherichia coli (E. coli) & 2 & $4.1 \%$ \\
Other & & \\
Enterococcus faecalis & 1 & $2.0 \%$ \\
Aeromonas hydrophila & 1 & $2.0 \%$ \\
Klebsiella pneumoniae & 1 & $2.0 \%$ \\
Combinations & & \\
MRSA, E. coli & 1 & $2.0 \%$ \\
MSSA, CoNS & 1 & $2.0 \%$ \\
Contaminants (n=17) & & \\
CoNS & 14 & $82.4 \%$ \\
MSSA & 1 & $5.9 \%$ \\
Streptococcus & 1 & $5.9 \%$ \\
Capnocytophaga & 1 & $5.9 \%$ \\
\hline
\end{tabular}

\section{Results}

During the study period, 738 joint aspirations were sent to the main hospital laboratory for testing, of which 358 aspirations in 348 patients met the inclusion criteria. The study group had a mean age of $57.8 \pm 17.3$ years and was comprised of approximately two-thirds male patients (Table 1). The most common joint aspirated was the knee (64.3\%). Nearly all aspirations were performed by either orthopaedic or emergency medicine physicians. Of the 358 aspirations, there were $49(13.7 \%)$ culture-positive aspirates for septic arthritis caused by 10 different strains of bacterial species (Table 2). Of the 49 cases of septic arthritis, 47 underwent surgical I\&D of the infected joint; one patient refused surgery; one patient underwent drain placement by interventional radiology. The most common infectious organisms were methicillin-sensitive (MSSA, 42.9\%) and methicillin-resistant (MRSA, 18.4\%) Staphylococcus aureus. Based on our definition, there were 17 cases of contaminants, of which six underwent surgical I\&D. Of the 17 cases of contaminants, 14 grew CoNS from the initial aspirate. The other three grew MSSA, Streptococcus, and Capnocytophaga species, but were successfully treated with conservative management based on low clinical suspicion of septic arthritis and were therefore deemed contaminants.

Of the 49 cases of septic arthritis, $30(61.2 \%)$ had a synovial $\mathrm{WBC} \geq 50,000 / \mathrm{mm}^{3}$, whereas 62 aseptic aspirations $(20.1 \%)$ had a synovial $\mathrm{WBC} \geq 50,000 / \mathrm{mm}^{3}$ $(\mathrm{p}<0.001)$, demonstrating an overall accuracy of $77.4 \%$ and a high NPV (Table 3). Of the 62 aseptic aspirations with synovial WBC $\geq 50,000 / \mathrm{mm}^{3}, 26$ $(41.9 \%)$ underwent surgical I\&D, of which 12 had crystals in the aspirate. Fourteen of the 49 cases of septic arthritis $(28.6 \%)$ had a positive synovial Gram stain compared to 3 of the 309 aseptic aspirations $(1.0 \%, \mathrm{p}<0.001)$, demonstrating an accuracy of $89.4 \%$ and high specificity (Table 3). Univariate analysis demonstrated that the septic arthritis group was younger $(49.7 \pm 18.7$ vs $59.1 \pm 16.7, \mathrm{p}<0.001)$ and had a greater rate of smokers $(44.0 \%$ vs $23.3 \%, p=0.002)$ compared to the aseptic group. The septic arthritis group presented with greater temperature $(99.2 \pm 1.5$ vs $\left.98.7 \pm 1.2^{\circ} \mathrm{F}, \mathrm{p}=0.045\right)$, ANC $(9.1 \pm 4.3$ vs $7.6 \pm 3.5$ $\left.\mathrm{x} 10^{3} / \mathrm{uL}, \quad \mathrm{p}=0.014\right), \quad \mathrm{ESR} \quad(76.1 \pm 31.7$ vs $59.3 \pm 35.6$ $\mathrm{mm} / \mathrm{hr}, \mathrm{p}=0.003)$, and CRP $(187.6 \pm 110.0$ vs $98.9 \pm 85.1$ $\mathrm{mg} / \mathrm{L}, \mathrm{p}<0.001)$ compared to the aseptic group, while serum WBC $\left(11.7 \pm 4.8\right.$ vs $\left.10.4 \pm 4.0 \times 10^{3} / \mathrm{uL}\right)$ trended toward statistical significance $(p=0.051)$. Multivariate regression analysis demonstrated that tobacco use $(p=0.001)$ and CRP $(p<0.001)$ remained significant risk factors/predictors for septic arthritis in the overall cohort.

Crystals were present in 163 aspirates (45.5\%, gout $n=115$, pseudogout $n=48$ ). Aspirates with crystals had a mean synovial WBC count of $48,612 / \mathrm{mm}^{3}$ with $91.4 \%$ PMNs, compared to $31,116 / \mathrm{mm}^{3}(\mathrm{p}=0.02)$ and $79.8 \%$ PMNs $(\mathrm{p}>0.001)$ in aspirates without crystals. Three joints $(0.8 \%$ overall rate) had concomitant crystalline arthropathy and septic arthritis, each of which had a synovial WBC $\geq 85,000 / \mathrm{mm}^{3} \quad$ (Table 4). Subgroup analysis demonstrated that in the crystal-positive cohort, the concomitant septic arthritis group demonstrated significantly greater serum WBC $(15.0 \pm 5.4$ vs $10.2 \pm 4.0$ $\left.\mathrm{x} 10^{3} / \mathrm{uL}, \quad \mathrm{p}=0.044\right)$ and synovial WBC count 
$\left(125,166 / \mathrm{mm}^{3}\right.$ vs $47,177 / \mathrm{mm}^{3}$, $\left.\mathrm{p}=0.017\right)$ compared to the aseptic group, although no patient factors or characteristics remained significant in the multivariate analysis. Of note, 47 of the 160 aseptic aspirates with crystals (29.4\%) had a synovial WBC $\geq 50,000 / \mathrm{mm}^{3}$. The accuracy of synovial WBC count as a diagnostic tool is greatly reduced in patients with crystalline arthropathy compared to patients without crystals $(71.2 \%$ vs $82.6 \%$ ) (Table 3$)$. This is further demonstrated in a ROC curve with an area under the curve (AUC) of 0.837 in patients without crystals compared to 0.705 in the overall patient group (Figure 1). Increasing the WBC count cutoff to $85,000 / \mathrm{mm}^{3}$ increased the specificity, although the PPV remained low (Table 3).

Eighty-two cases underwent surgical I\&D, of which 35 eventually had negative cultures (42.7\%). Of the 47 aseptic aspirates with crystals and synovial $\mathrm{WBC} \geq 50,000 / \mathrm{mm}^{3}, 12(25.5 \%)$ underwent surgical I\&D, which equaled $80 \%(12 / 15)$ of surgeries for aspirates with crystals and synovial WBC $\geq 50,000 / \mathrm{mm}^{3}$ (Table 5). Additionally, two patients with crystals and synovial WBC $<50,000 / \mathrm{mm}^{3}$ underwent surgery based on clinical presentation and presence of CoNS growth in the aspirate, which were later deemed to be contaminants.

ROC Curve for Synovial Cell Count and Septic Arthritis
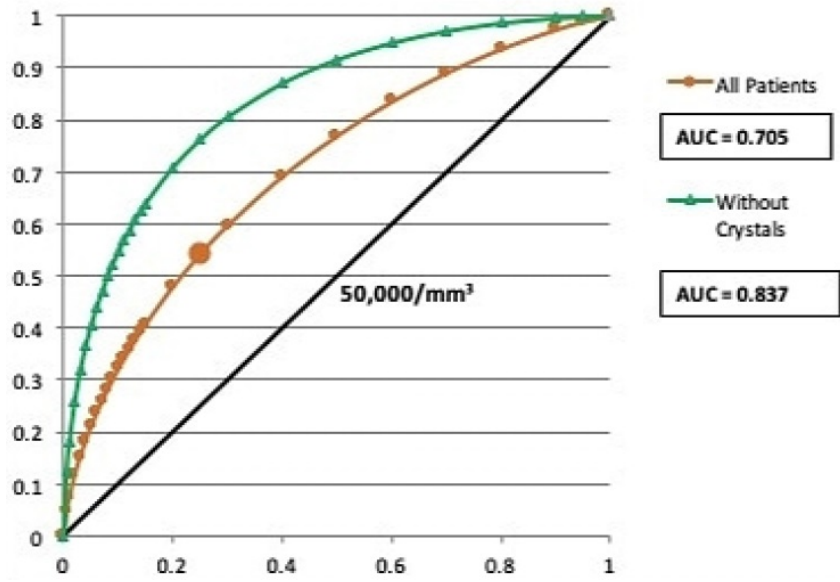

Figure 1. Receiver operating characteristics curve for synovial WBC cut-off of $50,000 / \mathrm{mm}^{3}$ in diagnosis of septic arthritis.

Table 3. Evaluation of synovial WBC count cut-off, gram stain, and presence of crystals in the diagnosis of septic arthritis. The sensitivity, specificity, positive predictive value (PPV), and negative predictive value (NPV) are presented for each test.

\begin{tabular}{|c|c|c|c|c|}
\hline & Septic joint & Aseptic joint & Total & \\
\hline \multicolumn{5}{|l|}{ Overall group } \\
\hline $\mathrm{WBC} \geq 50,000 / \mathrm{mm}^{3}$ & 30 & 62 & 92 & PPV: $32.6 \%$ (95\% CI 0.26-0.40) \\
\hline $\mathrm{WBC}<50,000 / \mathrm{mm}^{3}$ & 19 & 247 & 266 & NPV: $92.9 \%$ (95\% CI 0.90-0.95) \\
\hline \multirow[t]{2}{*}{ Total } & 49 & 309 & 358 & \\
\hline & Sensitivity: $61.2 \%$ (95\% CI 0.46-0.75) & Specificity: $79.9 \%$ (95\% CI 0.75-0.84) & & \\
\hline \multicolumn{5}{|l|}{ Overall group } \\
\hline Positive Gram stain & 14 & 3 & 17 & PPV: $82.4 \%$ (95\% CI 0.56-0.96) \\
\hline Negative Gram stain & 35 & 306 & 341 & NPV: $89.7 \%$ (95\% CI 0.89-0.91) \\
\hline \multirow[t]{2}{*}{ Total } & 49 & 309 & 358 & \\
\hline & Sensitivity: $28.6 \%$ (95\% CI 0.13-0.40) & Specificity: $99.0 \%$ (95\% CI 0.98-1.00) & & \\
\hline \multicolumn{5}{|c|}{$\begin{array}{l}\text { Aspirates with crystals (gout } n=115 \text {, } \\
\text { pseudogout } n=48 \text { ) }\end{array}$} \\
\hline $\mathrm{WBC} \geq 50,000 / \mathrm{mm}^{3}$ & 3 & 47 & 50 & PPV: $6.0 \%$ (95\% CI 0.05-0.08) \\
\hline $\mathrm{WBC}<50,000 / \mathrm{mm}^{3}$ & 0 & 113 & 113 & NPV: $100 \%$ \\
\hline \multirow[t]{2}{*}{ Total } & 3 & 160 & 163 & \\
\hline & Sensitivity: 100\% (95\% CI 0.29-1.0) & Specificity: 70.6\% (95\% CI 0.63-0.78) & & \\
\hline \multicolumn{5}{|c|}{ Aspirates without crystals } \\
\hline $\mathrm{WBC} \geq 50,000 / \mathrm{mm}^{3}$ & 27 & 15 & 42 & PPV: $64.3 \%$ (95\% CI 0.51-0.76) \\
\hline $\mathrm{WBC}<50,000 / \mathrm{mm}^{3}$ & 19 & 134 & 153 & NPV: $87.6 \%$ (95\% CI 0.83-0.91) \\
\hline \multirow[t]{2}{*}{ Total } & 46 & 149 & 195 & \\
\hline & Sensitivity: 58.7\% (95\% CI 0.43-0.73) & Specificity: 89.9\% (95\% CI 0.84-0.94) & & \\
\hline \multicolumn{5}{|c|}{$\begin{array}{l}\text { Aspirates with crystals (gout } n=115 \text {, } \\
\text { pseudogout } n=48 \text { ) }\end{array}$} \\
\hline $\mathrm{WBC} \geq 85,000 / \mathrm{mm}^{3}$ & 3 & 22 & 25 & PPV: $12.0 \%$ (95\% CI 0.10-0.14) \\
\hline $\mathrm{WBC}<85,000 / \mathrm{mm}^{3}$ & 0 & 138 & 138 & NPV: $100 \%$ \\
\hline \multirow[t]{2}{*}{ Total } & 3 & 160 & 163 & \\
\hline & Sensitivity: $100 \%$ (95\% CI 0.29-1.0) & Specificity: $86.3 \%$ (95\% CI 0.79-0.93) & & \\
\hline
\end{tabular}

Table 4. Characteristics of the three patients with concomitant crystalline and septic arthritis.

\begin{tabular}{|c|c|c|c|c|c|c|c|c|}
\hline Patient & Age & Sex & Joint & Gram stain & Synovial cell count & \% PMN & Crystals & Bacterial culture results \\
\hline 1 & 70 & Male & Knee & Positive & 115,500 & $98 \%$ & Gout & MSSA \\
\hline 2 & 86 & Male & Shoulder & Negative & 85,000 & $85 \%$ & Pseudogout & Pseudomonas \\
\hline 3 & 57 & Female & Ankle & Negative & 175,000 & $95 \%$ & Gout & S. sanguinis \\
\hline
\end{tabular}


Table 5. Breakdown of the 82 cases that underwent surgical irrigation and debridement with respect to presence of crystals and synovial WBC count.

\begin{tabular}{|c|c|c|c|c|c|}
\hline & $\mathrm{WBC} \geq 50 \mathrm{k} / \mathrm{mm}^{3}$, crystals & $\mathrm{WBC}<50 \mathrm{k} / \mathrm{mm}^{3}$, crystals & $W B C \geq 50 \mathrm{k} / \mathrm{mm}^{3}$, no crystals & $\mathrm{WBC}<50 \mathrm{k} / \mathrm{mm}^{3}$, no crystals & Total \\
\hline Septic & 3 & 0 & 26 & 18 & 47 \\
\hline Aseptic & 12 & 2 & 14 & 7 & 35 \\
\hline Total & 15 & 2 & 40 & 25 & 82 \\
\hline
\end{tabular}

\section{Discussion}

As the present study demonstrates, concomitant septic arthritis and crystalline arthropathy remains a rare entity. Given that both disease processes have similar presentations of joint swelling, pain, and erythema, differentiating between the two diseases presents a diagnostic dilemma. Our multivariate analysis of 358 joint aspirations found smoking status as a significant risk factor and elevated CRP $(>100$ $\mathrm{mg} / \mathrm{mL}$ ) as a significant predictor of septic arthritis. Nearly one-third of aseptic aspirates with crystalline arthropathy had synovial WBC $\geq 50,000 / \mathrm{mm}^{3}$, lowering the test's positive predictive value for septic arthritis to only $6 \%$. Conversely, aspirates without crystalline arthropathy had substantially greater PPV and accuracy when using synovial WBC count as diagnostic tool. The use of a cutoff of $50,000 / \mathrm{mm}^{3}$ as a diagnostic criterion to take a patient to the operating room resulted in 12 out of 15 patients with positive crystals undergoing a potentially unnecessary surgery to evacuate an aseptic joint. A cutoff of $85,000 / \mathrm{mm}^{3}$ improved specificity, although the PPV remained low, which is due to the low incidence of concomitant septic and crystalline arthropathy. Therefore, it is natural to conclude that in the setting of crystalline arthropathy, synovial cell count alone is not a reliable indicator for septic arthritis.

The infrequency of septic arthritis superimposed on crystalline arthropathy has previously been demonstrated with an incidence of 14 cases over 10 years at a large academic facility..$^{10}$ Similarly, Shah et al. ${ }^{8}$ revealed a $1.5 \%$ incidence of concurrent septic arthritis in the presence of crystals. This should not be interpreted as a protective effect of gout or pseudogout against pyogenic arthritis, as several studies have reported that patients with a history of crystalline arthropathy have underlying joint degeneration and multiple co-morbidities that make these patients more susceptible to develop a septic joint compared to patients without crystalline arthropathy. $8,13,14$

While most clinicians agree that synovial fluid analysis in patients with suspected septic arthritis should include Gram stain, culture, cell count with polymorphonuclear lymphocyte percentage, and crystal analysis, some have suggested synovial lactate, glucose, and leukocyte esterase, and real-time polymerase chain reaction (PCR) as adjunctive tools to aid the diagnosis of septic arthritis. ${ }^{15-19}$ The combination of leukocyte esterase and glucose strip testing of synovial fluid has a specificity of $99.2 \%$, making them valuable for ruling in as opposed to ruling out septic arthritis. ${ }^{17}$ Although available at our institution, leukocyte esterase and glucose strip testing is not part of our routine workup of septic arthritis. Currently, the leukocyte esterase strip test has been more widely accepted in the evaluation of periprosthetic joint infections rather than native joint infections. ${ }^{20}$ In our study, Gram stain was found to be the strongest rapid test for evaluating septic arthritis with a near- $100 \%$ specificity. As this is often the first test to result, it can be extremely valuable; however, with a sensitivity below 30\%, Gram stain, like the leukocyte esterase and glucose strip tests, is a poor screening test to rule out septic arthritis.

The receiver operating characteristics curve for synovial WBC count in our study suggests that a cut-off of $50,000 / \mathrm{mm}^{3}$ is an appropriate value to use in patients without crystals, but strictly abiding by this parameter would result in approximately $40 \%$ of patients with septic arthritis being misdiagnosed as aseptic, which is similar to previously reported rates, ${ }^{21}$ and would leave those patients untreated or undertreated. The accuracy of synovial cell count is greatly improved when patients with aspirates positive for crystals are filtered out, further highlighting the difficulty in diagnosing septic arthritis in the presence of synovial crystals. When synovial WBC count is applied to patients only with crystal-positive aspirates, the pitfalls associated with this test become apparent. While a sensitivity of $100 \%$ and a specificity of $70.6 \%$ would be considered very accurate for a medical test, the extreme rarity of concomitant gouty and septic arthritis changes the PPV from $64.3 \%$ in patients without crystals to just $6.0 \%$ in patients with crystals, a tenfold decrease. The false positives outweigh the true positives by a factor of $16: 1$, meaning that if $50,000 / \mathrm{mm}^{3}$ synovial WBC were the diagnostic threshold for septic arthritis, one would operate on 17 patients with gout to treat one patient with superimposed infection. This would lead to potentially unnecessary resource utilization, anesthesia, and surgical risk for 16 patients who could have been treated with medical management and observation pending culture results. 


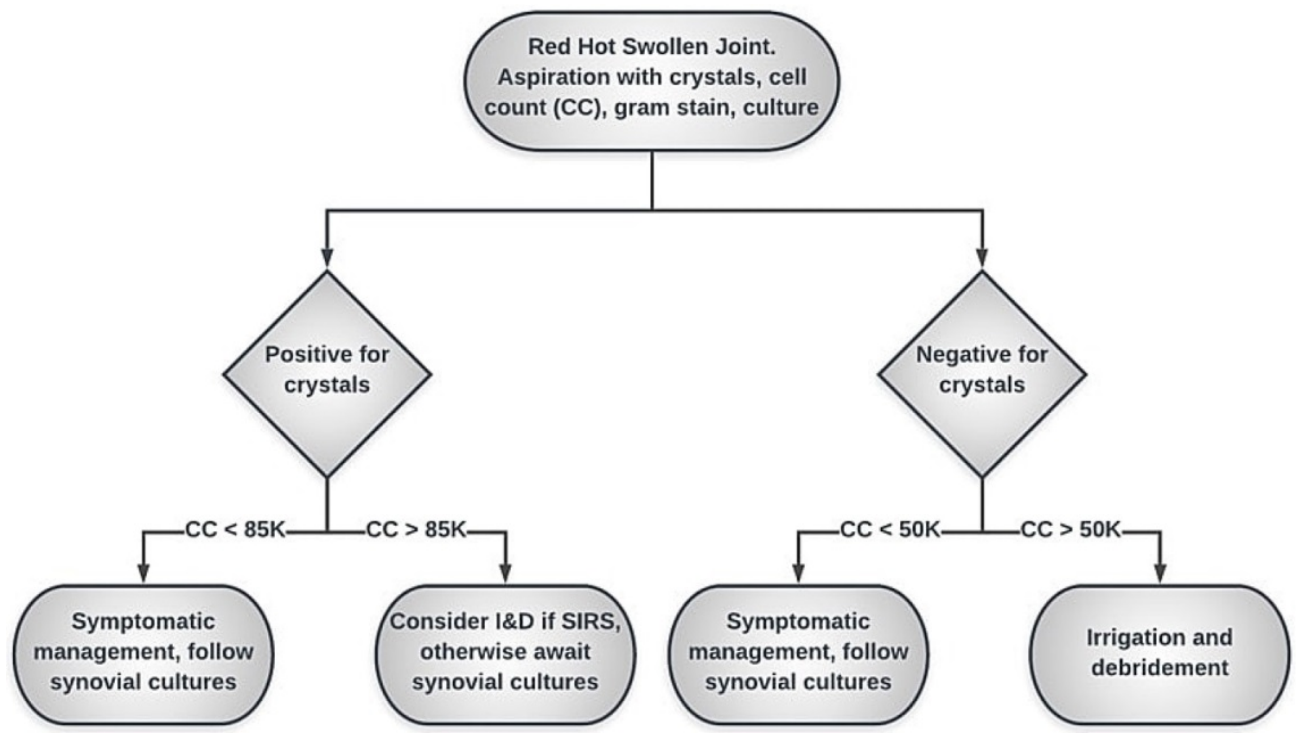

Figure 2. Flowchart illustrating our proposed algorithm using an increased cut-off of $85,000 / \mathrm{mm}^{3}$ in patients with crystalline arthropathy. Emergent surgery should be reserved for patients meeting criteria for systemic inflammatory response syndrome (SIRS) as a method of source control.

This study has several limitations, mainly owing to the possible administration of antibiotics prior to aspiration or intraoperative cultures, which affects the accuracy of the cell count and culture results. ${ }^{6,22}$ It is difficult to account for antibiotics administered at referring hospitals or by the primary medicine team prior to orthopaedic consultation and arthrocentesis due to the large number of transfers received at our tertiary care center; therefore, the true rate of concomitant septic and crystalline arthritis is likely greater than the three cases in this series. ${ }^{22}$ Our preferred strategy is to hold antibiotic treatment until intraoperative cultures are obtained. This also relates to the second limitation, the use of culture-dependent diagnosis rather than the use of Newman criteria, ${ }^{2}, 23$ which also considers turbid appearance of synovial fluid, pathogen isolated from a source other than synovial fluid, and histological findings. In our study, blood cultures were not routinely obtained to aid in diagnosis of septic arthritis; however, a positive blood culture in the setting of nebulous presentation may prompt surgical evacuation of the joint in question to eliminate a potential source of bacteremia.

In conclusion, synovial WBC cut-off of $50,000 / \mathrm{mm}^{3}$ in the setting of crystalline arthropathy is highly sensitive for superimposed septic arthritis, but lacks specificity, PPV, and accuracy in diagnosis. An increased cut-off of $85,000 / \mathrm{mm}^{3}$ may be more appropriate in patients with crystalline arthropathy, but still demonstrates a low PPV. Due to the rarity of concomitant septic and gouty arthritis, our proposed algorithm recommends medical management and observation in patients with crystal-positive joint aspirations unless the synovial WBC count is elevated above $85,000 / \mathrm{mm}^{3}$ (Figure 2). Emergent surgery should be reserved for patients with crystal-positive joint aspiration meeting criteria for systemic inflammatory response syndrome (SIRS) as a method of source control. Otherwise, we do not recommend routine antibiotic administration in the absence of positive synovial cultures. Surgical intervention may be delayed pending synovial culture results. Future, prospective studies using this treatment guideline are needed to evaluate its validity, accuracy and to assess patient outcomes.

\section{Acknowledgements}

TDL: data analysis, data interpretation, manuscript preparation; DLJ: study conception, data analysis, manuscript preparation; HBY: data collection, analysis, manuscript preparation; AZ: data interpretation, manuscript revision; SCT: manuscript revision; MKL: study conception, manuscript revision; JFP: study conception, data analysis, manuscript preparation/revision.

\section{Competing Interests}

The authors have declared that no competing interest exists.

\section{References}

1. Singh JA, Yu S. Septic Arthritis in Emergency Departments in the US: A National Study of Health Care Utilization and Time Trends. Arthritis Care Res (Hoboken). 2018;70:320-326.

2. Mathews CJ, Weston VC, Jones A, Field M, Coakley G. Bacterial septic arthritis in adults. Lancet. 2010;375:846-855.

3. Baker DG, Schumacher HR, Jr. Acute monoarthritis. N Engl J Med. 1993;329:1013-1020.

4. Lawrence RC, Helmick CG, Arnett FC, et al. Estimates of the prevalence of arthritis and selected musculoskeletal disorders in the United States. Arthritis Rheum. 1998;41:778-799.

5. Margaretten ME, Kohlwes J, Moore D, Bent S. Does this adult patient have septic arthritis? JAMA. 2007;297:1478-1488. 
6. McGillicuddy DC, Shah KH, Friedberg RP, Nathanson LA, Edlow JA. How sensitive is the synovial fluid white blood cell count in diagnosing septic arthritis? Am J Emerg Med. 2007;25:749-752.

7. Ross JJ. Septic Arthritis of Native Joints. Infect Dis Clin North Am. 2017;31:203-218.

8. Shah K, Spear J, Nathanson LA, McCauley J, Edlow JA. Does the presence of crystal arthritis rule out septic arthritis? J Emerg Med. 2007;32:23-26.

9. Schulz BM, Watling JP, Vosseller JT, Strauch RJ. Markedly elevated intra-articular white cell count caused by gout alone. Orthopedics. 2014;37:e739-742.

10. Weng CT, Liu MF, Lin LH, et al. Rare coexistence of gouty and septic arthritis: a report of 14 cases. Clin Exp Rheumatol. 2009;27:902-906.

11. Yu KH, Luo SF, Liou LB, et al. Concomitant septic and gouty arthritis--an analysis of 30 cases. Rheumatology (Oxford). 2003;42:1062-1066.

12. Fowler ML, Zhu C, Byrne K, et al. Pathogen or contaminant? Distinguishing true infection from synovial fluid culture contamination in patients with suspected septic arthritis. Infection. 2017;45:825-830.

13. Kennedy N, Chambers ST, Nolan I, et al. Native Joint Septic Arthritis: Epidemiology, Clinical Features, and Microbiological Causes in a New Zealand Population. J Rheumatol. 2015;42:2392-2397.

14. Lim SY, Lu N, Choi HK. Septic arthritis in gout patients: a population-based cohort study. Rheumatology (Oxford). 2015;54:2095-2099.

15. Shu E, Farshidpour L, Young M, Darracq M, Ives Tallman C. Utility of point-of-care synovial lactate to identify septic arthritis in the emergency department. Am J Emerg Med. 2019;37:502-505.

16. Lenski M, Scherer MA. Analysis of synovial inflammatory markers to differ infectious from gouty arthritis. Clin Biochem. 2014;47:49-55.

17. Omar M, Ettinger M, Reichling $M$, et al. Preliminary results of a new test for rapid diagnosis of septic arthritis with use of leukocyte esterase and glucose reagent strips. J Bone Joint Surg Am. 2014;96:2032-2037.

18. Mortazavi SMJ, Kalantar H, Baghdadi S, et al. The Utility of Leukocyte Esterase Strip Test in the Diagnosis of Pediatric Septic Arthritis. J Pediatr Orthop. 2019.

19. Yang S, Ramachandran P, Hardick A, et al. Rapid PCR-based diagnosis of septic arthritis by early Gram-type classification and pathogen identification. J Clin Microbiol. 2008;46:1386-1390.

20. Tischler EH, Cavanaugh PK, Parvizi J. Leukocyte esterase strip test: matched for musculoskeletal infection society criteria. I Bone Joint Surg Am. 2014;96:1917-1920.

21. Li SF, Henderson J, Dickman E, Darzynkiewicz R. Laboratory tests in adults with monoarticular arthritis: can they rule out a septic joint? Acad Emerg Med. 2004;11:276-280.

22. Ottink KD, Strahm C, Muller-Kobold A, Sendi P, Wouthuyzen-Bakker M. Factors to Consider When Assessing the Diagnostic Accuracy of Synovial Leukocyte Count in Periprosthetic Joint Infection. J Bone Jt Infect. 2019;4:167-173.

23. Newman JH. Review of septic arthritis throughout the antibiotic era. Ann Rheum Dis. 1976;35:198-205. 